\title{
LA PLANEACION ESTRATEGICA COMO HERRAMIENTA PARA MEJORAR LA PRODUCTIVIDAD EN LAS AGENCIAS DE VIAJES Y TURISMO DE LIMA
}

\author{
STRATEGIC PLANNING AS A TOOL TO IMPROVE PRODUCTIVITY \\ IN THE TRAVEL AGENCIES OF LIMA \\ Jorge Vicente Mayurí Barrón * \\ Docente Asociado de la Facultad de Ciencias Administrativas - UNMSM \\ [Recepción: Octubre de 2011/ Conformidad: Noviembre de 2011]
}

\section{RESUMEN}

El uso de la Planeación Estratégica como herramienta a través de sus matrices permite que este estudio de aplicación para las Agencias de Viajes y Turismo mejoren su productividad debido a la alta competencia en el mercado y así mismo por la reducción de comisiones por parte de las aerolíneas nacionales e internacionales y algunos prestatarios de la industria turística, obligando a mejorar la calidad de gestión por parte de las Agencias de Viajes y Turismo.

Las estadísticas obtenidas a través del MINCETUR (Ministerio de Comercio Exterior y Turismo) determinaron un total de 2,383 agencias existentes en la ciudad de Lima de las cuales se ha tomado como referencia 10 de las mejores agencias que operan turismo receptivo, agresivo e interno, y que al mismo tiempo son agentes de pasaje IATA y en algunos casos operan como tour operadoras. Se demuestra en los resultados que 7 de las 10 agencias manejan Planeamiento Estratégico, y que por esta razón llegaron a incrementar su productividad.

Palabras Claves: Planeación, Productividad, Agencia De Viajes, Turismo

\begin{abstract}
The use of Strategic Planning as a tool through its matrix allows this study to be applied to Travel Agencies to improve their productivity due to high competition in the market and also for the reduction of commissions from national and international airlines, and some tourism industry borrowers, forcing to improve the quality of management by travel agencies.

Statistics obtained through MINCETUR (Ministry of Foreign Trade and Tourism) identified a total of 2.383 existing travel agencies in the city of Lima taking as reference 10 of the best travel agencies operating inbound tourism, domestic and international at the same time, the travel agencies are IATA Passenger Agents and sometimes operating as tour operators.

Results demonstrates that 7 of 10 agencies handle strategic planning, and for this reason they came to increase productivity
\end{abstract}

Keywords: Planning, Productivity, travel agencies, tourism

* Doctor en Educación (UNMSM), Magister en Administración, con Mención en Gestión Empresarial (UNMSM); Licenciado en Administración de Empresas (Universidad Ricardo Palma) Post Grado en Derecho y Espacial (UNMSM), Profesor Asociado de la Facultad de Ciencias Administrativas de la UNMSM. 


\section{INTRODUCCION}

Las Agencias de Viaje y Turismo en nuestro país pertenecen a un sector de enorme importancia para la economía de la nación. De ella se extrae el 10\% del PBI, siendo una fuente generadora de recursos innegable.

La apertura y creación de nuevos destinos turísticos en nuestro país, y los Tratados de Libre Comercio y la globalización del mercado, hacen que el Perú en muchas de sus regiones sea un destino turístico único haciendo que muchas micro y pequeñas empresas se desarrollen en este rubro.

Dada la alta competencia, es necesario que la gran mayoría de ellas empiecen a utilizar herramientas no solo de sobrevivencia a corto plazo, sino de competencia para existir y ofrecer mejores productos, tal es el caso de La Planeación estratégica.

Toda empresa diseña planes estratégicos para el logro de sus objetivos y metas planteadas, esto planes pueden ser a corto, mediano y largo plazo, según la amplitud y magnitud de la empresa. Es decir, su tamaño, ya que esto implica que cantidad de planes y actividades debe ejecutar cada unidad operativa, ya sea de niveles superiores o niveles inferiores.

Ha de destacarse que el presupuesto refleja el resultado obtenido de la aplicación de los planes estratégicos, es de considerarse que es fundamental conocer y ejecutar correctamente los objetivos para poder lograr las metas trazadas por las empresas.

También es importante señalar que la empresa debe precisar con exactitud y cuidado la misión que se va regir la empresa, la misión es fundamental, ya que esta representa las funciones operativas que va a ejecutar en el mercado y va a suministrar a los consumidores.

\section{PLANIFICACIÓN ESTRATÉGICA}

Constituye un sistema gerencial que desplaza el énfasis en el "qué lograr" (objetivos) al "qué hacer" (estrategias) Con la Planificación Estratégica se busca concentrarse en sólo, aquellos objetivos factibles de lograr y en qué negocio o área competir, en correspondencia con las oportunidades y amenazas que ofrece el entorno.

Hace falta impulsar el desarrollo cultural, esto significa que todas las personas relacionadas con la organización se desarrollen en su saber, en sus expectativas, en sus necesidades, y en sus formas de relacionarse y de enfrentar al mundo presente y futuro, esencialmente dinámico.

Se consideran cuatro puntos de vista en la Planeación Estratégica:

\section{EL PORVENIR DE LAS DECISIONES ACTUALES}

Primero, la planeación trata con el porvenir de las decisiones actuales. Esto significa que la planeación estratégica observa la cadena de consecuencias de causas y efectos durante un tiempo, relacionada con una decisión real o intencionada que tomará el director. La esencia de la planeación estratégica consiste en la identificación sistemática de las oportunidades y peligros que surgen en el futuro, los cuales combinados con otros datos importantes proporcionan la base para qué una empresa tome mejores decisiones en el presente para explotar las oportunidades y evitar los peligros. Planear significa diseñar un futuro deseado e identificar las formas para lograrlo.

\section{Proceso}

Segundo, la planeación estratégica es un proceso que se inicia con el establecimiento de 
metas organizacionales, define estrategias y políticas para lograr estas metas, y desarrolla planes detallados para asegurar la implantación de las estrategias y así obtener los fines buscados. También es un proceso para decidir de antemano qué tipo de esfuerzos de planeación debe hacerse, cuándo y cómo debe realizarse, quién lo llevará a cabo, y qué se hará con los resultados. La planeación estratégica es sistemática en el sentido de que es organizada y conducida con base en una realidad entendida.

Para la mayoría de las empresas, la planeación estratégica representa una serie de planes producidos después de un periodo de tiempo específico, durante el cual se elaboraron los planes. También debería entenderse como un proceso continuo, especialmente en cuanto a la formulación de estrategias, ya que los cambios en el ambiente del negocio son continuos. La idea no es que los planes deberían cambiarse a diario, sino que la planeación debe efectuarse en forma continua y ser apoyada por acciones apropiadas cuando sea necesario.

\section{Filosofía}

Tercero, la planeación estratégica es una actitud, una forma de vida; requiere de dedicación para actuar con base en la observación del futuro, y una determinación para planear contante y sistemáticamente como una parte integral de la dirección. Además, representa un proceso mental, un ejercicio intelectual, más que una serie de procesos, procedimientos, estructuras o técnicas prescritos.

\section{Estructura}

Cuarto, un sistema de planeación estratégica formal une tres tipos de planes fundamentales, que son: planes estratégicos, programas a mediano plazo, presupuestos a corto plazo y planes operativos. La planeación estratégi- ca es el esfuerzo sistemático y más o menos formal de una compañía para establecer sus propósito, objetivos, políticas y estrategias básicas, para desarrollar planes detallados con el fin de poner en práctica las políticas y estrategias y así lograr los objetivos y propósitos básicos de la compañía.

\section{LO QUE NO ES LA PLANEACIÓN ESTRATÉGICA}

La planeación estratégica no trata de tomar decisiones futuras, ya que éstas sólo pueden tomarse en el momento. La planeación del futuro exige que se haga la elección entre posibles sucesos futuros, pero las decisiones en sí, las cuales se toman con base en estos sucesos, sólo pueden hacerse en el momento.

La planeación estratégica no pronostica las ventas de un producto para después determinar qué medidas tomar con el fin de asegurar la realización de tal pronóstico en relación con factores tales como: compras de material, instalaciones, mano de obra, etc.

La planeación estratégica no representa una programación del futuro, ni tampoco el desarrollo de una serie de planes que sirvan de molde para usarse diariamente sin cambiarlos en el futuro lejano. Una gran parte de empresas revisa sus planes estratégicos en forma periódica, en general una vez al año. La planeación estratégica debe ser flexible para poder aprovechar el conocimiento acerca del medio ambiente.

La planeación estratégica no representa esfuerzo para sustituir la intuición y criterio de los directores.

La planeación estratégica no es nada más un conjunto de planes funcionales o una extrapolación de los presupuestos actuales; es un enfoque de sistemas para guiar una empresa durante un tiempo a través de su medio ambiente, para lograr las metas dictadas.

Modelos conceptuales de la planeación estratégica. 
Un modelo conceptual es aquel que presenta una idea de lo que algo debería ser en general, o una imagen de algo formado mediante la generalización de particularidades. A cambio, un modelo operativo es el que se usa en realidad en las empresas.

\section{LAS PRINCIPALES}

CARACTERÍSTICAS DE LOS MODELOS CONCEPTUALES DE LA PLANEACIÓN ESTRATÉGICA SON

Antes de llevar a cabo un programa estratégico de planeación es importante que las personas involucradas en él tengan un amplio conocimiento de lo que tiene en mente el alto directivo y cómo operará el sistema. Esta guía está incorporada en un plan para planear, el cual puede ser oral, aunque usualmente es escrito, para su distribución general.

La información acumulada en estas áreas algunas veces es llamada "análisis de situación”, pero también se usan otros términos para denominar esta parte de la planeación.

Ninguna organización, no importa cuan grande o lucrativa sea, puede examinar en forma minuciosa todos los elementos que posiblemente están incluidos en el análisis de la situación. Es por este que cada organización debe identificar aquellos elementos - pasados, presentes y futuros -, que son de gran importancia para su crecimiento, prosperidad y bienestar, y debe concentrar su pensamiento y sus esfuerzos para entenderlos. Otros elementos se pueden considerar en esta parte del proceso de la planeación aunque pueden ser estimados sin ser investigados o sacados de documentos publicados al respecto.

\section{Formulación De Planes}

En cuanto a conceptos y con base en las premisas antes mencionadas, el siguiente paso en el proceso de planeación estratégica es formular estrategias maestras y de progra- mas. Las estrategias maestras se definen como misiones, propósitos, objetivos y políticas básicas; mientras que las estrategias de programa se relacionan con la adquisición, uso y disposición de los recursos para proyectos específicos, tales como la construcción de una nueva planta en el extranjero.

A diferencia de la programación a mediano plazo no existe un enfoque modelo para planear en esta área. Lo que se hace depende de los deseos de los directores en un momento dado, los cuales, a su vez, son estimulados por las condiciones a las que se enfrenta la empresa en un momento preciso.

La programación a mediano plazo es el proceso mediante el cual se prepara y se interrelacionan planes específicos funcionales para mostrar los detalles de cómo se debe llevar a cabo le estrategia para lograr objetivos, misiones y propósitos de la compañía a largo plazo. El periodo típico de planeación es de cinco años, pero existe una tendencia en las compañías más avanzadas en cuanto a tecnología, de planear por adelantado de siete a diez años. Las empresas que se enfrentan a ambientes especialmente problemáticos algunas veces reducen la perspectiva de planeación a cuatro o tres años.

El siguiente paso es desarrollar los planes a corto plazo con base en los planes a mediano plazo. En algunas organizaciones los números obtenidos durante el primer año de los planes a mediano plazo son los mismos que aquellos logrados con los planes operativos anuales a corto plazo, aunque en otras empresas no existe la misma similitud. Los planes operativos serán mucho más detallados que los planes de programación a mediano plazo.

\section{Implementación Y Revisión}

Una vez que los planes operativos son elaborados deben ser implantados. El proceso de implantación cubre toda la gama de activi- 
dades directivas, incluyendo la motivación, compensación, evaluación directiva y procesos de control.

Los planes deben ser revisados y evaluados. No existe mejor manera para producir planes por parte de los subordinados que cuando los altos directivos muestran un interés profundo en éstos y en los resultados que pueden producir.

Cuando fue desarrollada pro primera vez la planeación formal en la década de los cincuenta, las compañías tendían a hacer planes por escrito y no revisarlos hasta que obviamente eran obsoletos. En la actualidad, la gran mayoría de las empresas pasa por un ciclo anual de planeación, durante el cual se revisan los planes. Este proceso debería contribuir significativamente al mejoramiento de la planeación del siguiente ciclo.

\section{FLUJOS DE INFORMACIÓN Y NORMAS DE EVALUACIÓN Y DECISIÓN}

Los "flujos de información", simplemente deben transmitir el punto de que la información "fluye" por todo el proceso de planeación. Este "flujo" difiere grandemente, dependiendo de la parte del proceso a la que sirve y del tema de la información.

En todo proceso de planeación es necesario aplicar las normas de decisión y evaluación. Por otra parte, con el desarrollo de los planes operativos actuales, las normas de decisión se convierten en más cuantitativas, o sea, en fórmulas de sustitución de inventarios o de rendimientos sobre inversión.

La planificación Estratégica es una herramienta por excelencia de la Gerencia Estratégica, consiste en la búsqueda de una o más ventajas competitivas de la organización y la formulación y puesta en marcha de estrategias permitiendo crear o preservar sus ventajas, todo esto en función de la Misión y de sus objetivos, del medio ambiente y sus presiones y de los recursos disponibles.

Sallenave (1991), afirma que "La Planificación Estratégica es el proceso por el cual los dirigentes ordenan sus objetivos y sus acciones en el tiempo. No es un dominio de la alta gerencia, sino un proceso de comunicación y de determinación de decisiones en el cual intervienen todos los niveles estratégicos de la empresa”.

La Planificación Estratégica tiene por finalidad producir cambios profundos en los mercados de la organización y en la cultura interna.

La expresión Planificación Estratégica es un Plan Estratégico Corporativo, el cual se caracteriza fundamentalmente por coadyuvar a la racionalización de la toma de decisiones, se basa en la eficiencia institucional e integra la visión de largo plazo (filosofía de gestión), mediano plazo (planes estratégicos funcionales) y corto plazo (planes operativos).

Es el conjunto de actividades formales encaminadas a producir una formulación estratégica. Estas actividades son de muy variado tipo y van desde una reunión anual de directivos para discutir las metas para el ejercicio entrante, hasta la obligatoria recopilación y envío de datos presupuestarios por parte de todas las unidades de la empresa a la unidad superior. Son aquellos mecanismos formales, es decir, de obligado cumplimiento a plazo fijo, que "fuerzan" el desarrollo de un plan estratégico para la empresa.

Aporta una metodología al proceso de diseño estratégico, guían a la dirección en la tarea de diseñar la estrategia.

La planificación estratégica no es sólo una herramienta clave para el directivo implica, necesariamente, un proceso i nter-activo de arriba abajo y de abajo arriba en la orga- 
nización; la dirección general marca metas generales para la empresa (apoyada en la información de mercados recibida, con seguridad, de las unidades inferiores) y establece prioridades; las unidades inferiores determinan planes y presupuestos para el período siguiente; esos presupuestos son consolidados y corregidos por las unidades superiores, que vuelven a enviarlos hacia abajo, donde son nuevamente retocados, etc. Como consecuencia, el establecimiento de un sistema formal de planificación estratégica hace descender la preocupación estratégica a todos los niveles de la organización.

\section{PLANEACIÓN ESTRATÉGICA}

La empresa selecciona, entre varios caminos alternativos, el que considera más adecuado para alcanzar los objetivos propuestos. Generalmente, es una planeación global a largo plazo.

La planeación estratégica exige cuatro fases bien definidas: formulación de objetivos organizacionales; análisis de las fortalezas y limitaciones de la empresa; análisis del entorno; formulación de alternativas estratégicas.

Planificación estratégica: utilización del proceso.

La planeación estratégica: es el proceso administrativo de desarrollar y mantener una relación viable entre los objetivos recursos de la organización y las cambiantes oportunidades del mercado. El objetivo de la planeación estratégica es modelar y remodelar los negocios y productos de la empresa, de manera que se combinen para producir un desarrollo y utilidades satisfactorios.

La planificación estratégica proporciona la dirección que guiará la misión, los objetivos y las estrategias de la empresa, pues facilita el desarrollo de planes para cada una de sus áreas funcionales. Un plan estratégi- co completo guía cada una de las áreas en la dirección que la organización desea seguir y les permite desarrollar objetivos, estrategias y programas adecuados a las metas. La relación entre la planificación estratégica y la de operaciones es parte importante de las tareas de la gerencia.

\section{AGENCIAS DE VIAJES Y TURISMO}

Toda Agencia de Viajes y Turismo independientemente de su clasificación debe de someterse al Decreto Supremo $\mathrm{N}^{\circ}$ 026-2004-MINCETUR el cual es el Reglamento de Agencias de Viajes y Turismo, este establece los requisitos y procedimientos para autorizar la prestación del servicio de Agencia de Viajes y Turismo, define el concepto de Agencias de Viajes y Turismo, establece las condiciones que deberán cumplir para la prestación del servicio. Establece la Clasificación como: Minorista, Mayorista y Operador de Turismo, y precisa la obligatoriedad de presentar una Declaración Jurada que de cuenta el cumplimiento de requisitos mínimos. Entre ellas se menciona la adecuada organización y planificación de sus metas.

\section{DEL ESTUDIO PROYECTADO}

Se ha estimado un incremento del $15 \%$ en la comercializacion de los servicios turisticos por parte de las Agencias de Viajes y Turismo que implementan la Planeacion Estrategica en su gestion, y los resultados arrojan un resultado mayor en 2.5 puntos.

El numero de Agencias de Viajes y Turismo, es pequeña, dado que la mayoría de ellas se encuentran en diferentes provincias o en los conos. Dado que no se ha utilizado encuestas, sino se determino entrevistar directamente a los directivos, en algunos casos las Agencias de Viajes y Turismo, no querian 
dar información cuantitativa de esa naturaleza, por lo que se trabajo con datos porcentuales.

Acorde a los objetivos del estudio, se ha determinado que estas Agencias de Viaje y Turismo, mejoran sus indices de productividad traducido en utilidades y aumento de clientes gracias al uso de herramientas de gestion empresarial de Planeación Estrategica.

\section{CONCLUSIONES}

La Planeación Estratégica mejora la productividad en las Agencias de Viaje y Turismo La prosperidad de Agencias de Viaje y Turismo se ven reflejados en la llegada de pasajeros nacionales e internacionales acorde a los datos emitidos por el Ministerio de Interior (Migraciones) y el MINCETUR

\section{REFERENCIAS BIBLIOGRAFICAS}

1. ACKOFF, Russell L. (1995): "Planificación De La Empresa Del Futuro”. México. Editorial Limusa S.A. De C.V.

2. BATEMAN, Thomas S. (2005): “Administración: Un Nuevo Panorama Competitivo" México, D.F. Mcgraw-Hill.

3. BATEMAN, Thomas S. (2009): "Administración: Liderazgo Y Colaboración En Un Mundo Competitivo". México, D.F.: Mcgraw-Hill.

4. BERNAL TORRES, César A. (2007): "Introducción A La Administración De Las Organizaciones: Enfoque Global E Integra”. México, D.F.: Pearson.

5. BONIFACE, Priscilia. (1995): "Managing Quality Cultural Tourism”. Routledge London/New York.

6. BURNS, Peter; HOLDEN, Andrew. (1995): "Tourism: A New Perspective". Prentice hall. London.

7. CERTO, Samuel / PETER, J.Paul / OTTENSMEYER, Edward. (1996): "Dirección Y Administración Estratégicas”. 3era. Ed. Rev. Corregida Y Aumentada Madrid /
Mosby-Doyma Libros, S.A. -División Irwin.

8. CERTO, Samuel C. (2001): "Administración Moderna: Diversidad, Calidad, Ética, \& El Entorno Global”. Bogotá. Prentice Hall.

9. CHIAVENATO, Adalberto (2007): "Introducción A La Teoría General De La Administración”. México, D.F.: Mcgraw-Hil.

10. DAVID, Fred D. (2008): "Conceptos De Administración Estratégica”. México, D.F.: Pearson, 11 edicion.

11. DRUKER, Peter (2006). "Druker Para Todos Los Días: 366 Días De Reflexiones Clave Para Acertar En Sus Negocios". Bogota: Editorial Norma .

12. DRUKER, Peter. (2003): "La Gerencia En La Sociedad Futura”. Colombia. Editorial Norma S.A.

13. GARTNER, William C. (1996): "Tourism Development, Principles, Processes And Policies". New York : Van Nostrand Reinhold.

14. GEE, Chuck Y. (1997): "The Travel Industry". 3a. ed. Van Nostrand Reinhold. New York.

15. HARRISON, Lynn C. (1996): "Practicing Responsible Tourism. International Case Studies In Tourism Planning, Policy And Development". Wiley. New York.

16. HERNÁNDEZ y RODRÍGUEZ, Sergio (2008): "Administración: Teoría, Proceso, Áreas Funcionales Y Estrategias Para La Competitividad". México, D. F.: McgrawHill.

17. HILL, Charles W.L. / JONES Gareth R. (1996): "Administración Estratégica, Un Enfoque Integrado”. 3era. Edición Ampliada. Mc Graw-Hill Interamericana, S.A.

18. HITT, Michael A. (2008): "Administración Estratégica: Competitividad Y Globalización. Conceptos Y Caso". México, D.F.: Thomson.

19. HITT, Michael A., Black, J. Stewart Y Porter, Lyman W. (2006): “Administración”. México, D. F.: Pearson.

20. JONES, Gareth R. (2006): "Administración Contemporánea”. México, D.F.: McgrawHil. 